\section{Cardiac Angiosarcoma and Hepatic Hemangioma}

Kazuyoshi Tanigawa, MD; Hiroaki Kawano, MD; Tomayoshi Hayashi, MD; Tadashi Hasegawa, MD; Koji Maemura, MD; Kiyoyuki Eishi, MD

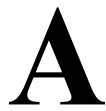
62-year-old-Japanese man was admitted to our hospital because of a cardiac tumor. He had no symptoms. Magnetic resonance image performed for the precise evaluation of the hepatic tumor revealed a cardiac tumor in the atrial septum in addition to a hepatic tumor (Figure A,B). Those tumors did not connect with each other (Figure C). Chest X-ray and electrocardiography were normal. Transthoracic echocardiography revealed a large mass protruding into the bilateral atria (right atrium, $3.8 \times 3.5 \times 3.2 \mathrm{~cm}$; left atrium, $3.4 \times 3.4 \times 2.7 \mathrm{~cm}$ ) (Figure D). Laboratory data were normal. Coronary angiography demonstrated tumor-staining (Figure $\mathbf{E}$ ). The cardiac tumor was completely resected 1 week after admission (Figure $\mathbf{F}, \mathbf{G}$ ). Histologically, large atypical cells formed lumen and irregular anastomosing vascular networks (Figure H,I). Immunohistochemically, those cells were positive for CD31 (Figure J), CD34 (Figure K), and Factor VIII (Figure L). Therefore, this tumor was diagnosed as cardiac angiosar- coma. Seven weeks after the operation, the hepatic tumor was resected. Pathological examination indicated typical cavernous hemangioma (Figure M). No additional treatment was decided at the meeting with oncologists.

As a coexisting cardiac angiosarcoma and hepatic hemangioma is very rare, this patient should be observed carefully.

\section{Disclosures}

K.M. is a member of Circulation Journal's Editorial Team. The authors have no conflicts of interest to declare.

Received December 3, 2020; revised manuscript received December 8, 2020; accepted December 10, 2020; J-STAGE Advance Publication released online February 4, 2021 Time for primary review: 4 days

Department of Cardiovascular Surgery (K.T., K.E.), Department of Cardiovascular Medicine (H.K., K.M.), Nagasaki University Graduate School of Biomedical Sciences, Nagasaki; Department of Pathology, Nagasaki Prefecture Shimabara Hospital, Shimabara (T. Hayashi); and Department of Surgical Pathology, Sapporo Medical University School of Medicine, Sapporo (T. Hasegawa), Japan

Mailing address: Hiroaki Kawano, MD, Department of Cardiovascular Medicine, Nagasaki University Graduate School of Biomedical Sciences, 1-7-1 Sakamoto, Nagasaki 852-8501, Japan. E-mail: hkawano@nagasaki-u.ac.jp

All rights are reserved to the Japanese Circulation Society. For permissions, please e-mail: cj@j-circ.or.jp ISSN-1346-9843
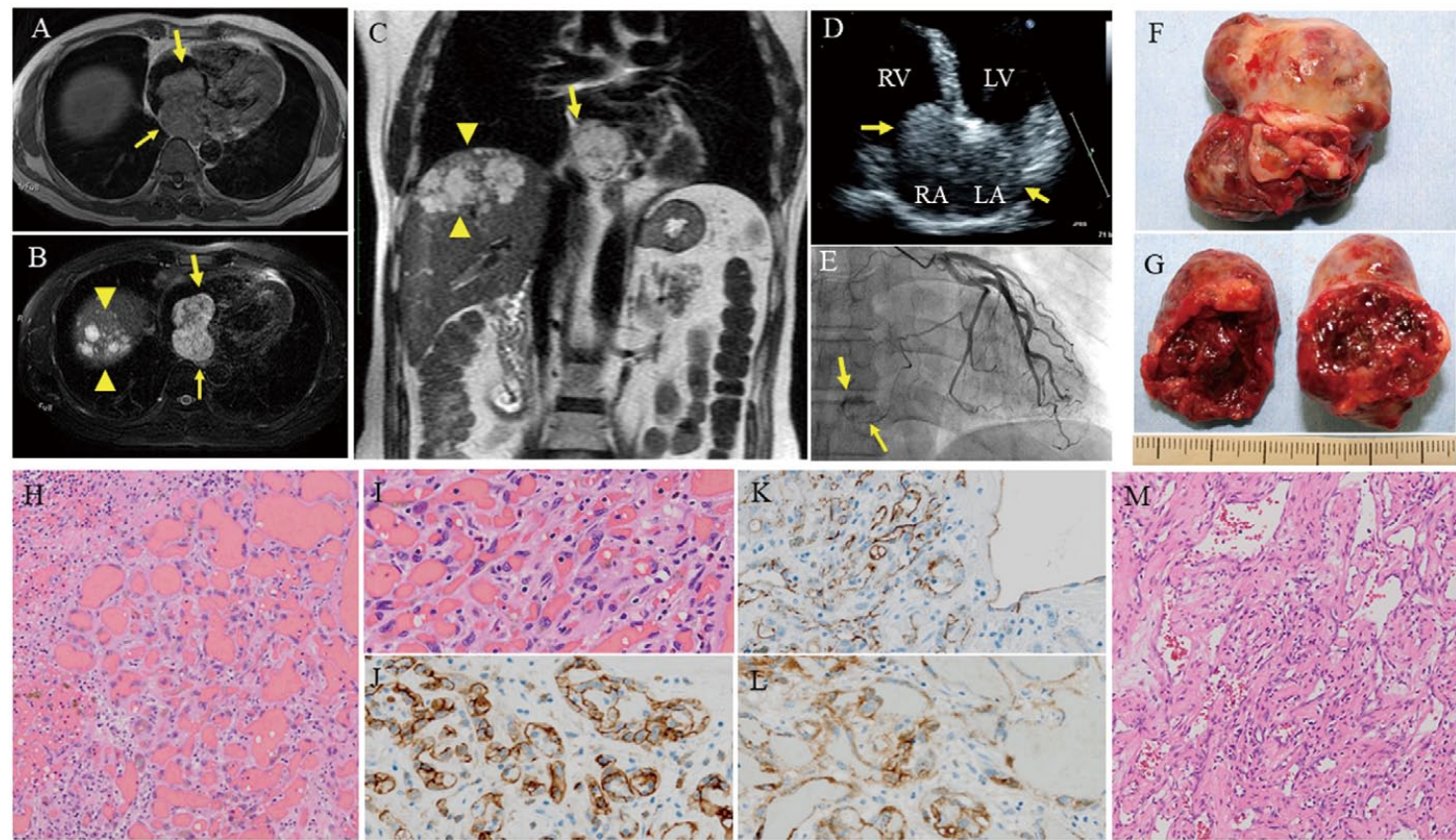

Figure. Magnetic resonance image demonstrates a cardiac tumor (arrows) and a hepatic tumor (arrow heads) (A, T1-weighed image; B and C, T2-weighed image). Transthoracic echocardiography shows a cardiac mass (D; RA, right atrium; LA, left atrium; $\mathrm{RV}$ right ventricle; LV, left ventricle). Coronary angiography shows tumor staining (E). The tumor is dumbbell-shaped $(\mathbf{F})$ and the inside is red $(\mathbf{G})$. Histopathology shows cardiac angiosarcoma [hematoxylin and eosin (H,I); immunostaining for CD31 (J), CD34 $(\mathbf{K})$, and Factor VIII (L)], and hepatic cavernous hemangioma (M). 